\title{
Influence of Charge Injection on the Formation of Remanent Polarization in P(VDF-TrFE) Copolymers
}

\author{
E. Bihler, G. Neumann, G. Eberle, W. Eisenmenger \\ 1. Physikalisches Institut, Universität Stuttgart, \\ Stuttgart, Germany
}

\begin{abstract}
The influence of charge injection on the formation of remanent polarization in $\mathrm{P}$ (VDF-TrFE) copolymers is studied by inserting an insulating barrier between the metal electrodes and the P(VDF-TrFE) copolymer during the poling process. The resulting remanent polarization is significantly smaller than that of identically poled samples with the same field strength and time duration applied, but with directly contacting metal electrodes. This shows that the orientation of the dipoles alone is not a sufficient condition for the formation of remanent polarization in PVDF and its copolymers. Our experiments indicate that the stabilization of the polarization is mediated by charge injection and charge trapping at polarized crystallite surfaces.
\end{abstract}

\section{Introduction}

The origin of the high stability of the polarization in poled films of PVDF and its copolymers with TrFE are still open to discussion.

The observation of spontaneous polarization and dipole rotation in the nearhexagonal lattice of the polar $\beta$-crystallites in $60^{\circ}$ steps [5] gives evidence for ferroelectricity in the polar crystallites of PVDF.

By poling the dipoles are rotated into the direction of the applied field and are kept in this orientation after switching off the poling field. The dipole orientation in the polar crystallites is considered to be stabilized by the anisotropy field of the near-hexagonal lattice.

Since the poling needs high electric fields $(\mathrm{MV} / \mathrm{cm})$ charge injection at the electrodes becomes possible. The role of these injected space charges for the piezoelectricity observed in poled PVDF films has been discussed extensively in the literature (see for a review [1]).

In order to investigate the importance of charge injection for the formation of piezoelectricity in the films, poling experiments have been performed with blocking electrodes $[2,3,4]$. Usually the films are coated with evaporated metal electrodes for poling. The charge injection from the metal electrode into the film can be hindered by inserting a thin insulating layer between the metal electrode and the PVDF film. 
In all previous experiments with blocking electrodes the films have been poled at elevated temperature $\left(80-100^{\circ} \mathrm{C}\right)$ and at rather low field strength $(0.3$ $0.4 \mathrm{MV} / \mathrm{cm}$ ) for longer times (i.e., hours). A direct comparison of the resulting remanent polarizartion after poling with contacting and blocking electrodes, respectively, is not possible under these conditions since the conductivity of PVDF is at least two orders of magnitude larger than that of the blocking insulator. Therefore after applying a voltage step to the sandwich sample: insulator/PVDF film/insulator the voltage across the PVDF sample decays within a few $100 \mathrm{~s}$.

\section{Experimental}

Figure 1 shows the electric diagram of the sample sandwich consisting of blocking insulating layers and the PVDF film.

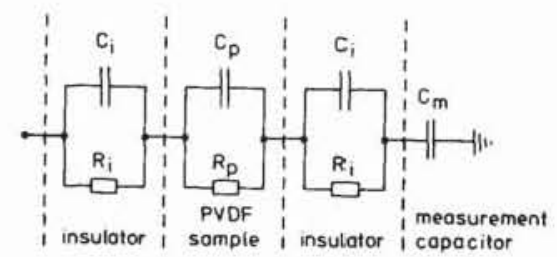

insulator somple insulator icapacitor
Figure 1: Electric diagram of the sample sandwich. The electric properties of the materials are characterized by a capacitance and a resistor in parallel. The voltage at capacitor $C_{m}$ is a measure for the dielectric displacement during poling.

The relation between the voltage drop across the PVDF sample and the insulator can be estimated for two cases:

1. For times short compared to the charge relaxation times in the blocking layers $\tau_{i}=R_{i} C_{i}$ and in the sample $\tau_{p}=R_{p} C_{p}$, respectively, the voltages are determined by the capacitances $C_{p}$ ans $C_{i}$. This condition is limited by the charge relaxation time $\tau_{p}$ of PVDF which is $\approx 100 \mathrm{~s}$. During that time the dielectric displacement is the same in all materials.

2. For times longer than the relaxation times the voltage drop is determined by the resistors $R_{i}$ and $R_{p}$. Since the blocking electrode has a large resistance compared to the PVDF sample, the voltage drop in this case develops only across the blocking insulator films.

Consequently the poling time must be short enough to ensure that the full potential is applied to the PVDF sample. In addition the applied potential must be large enough to polarize the PVDF film. A limitation is given by the dielectric strength of the insulating material. The dielectric displacement is constant through all materials. Therefore the insulator will be polarized to the same extent as the PVDF film. Most insulators cannot withstand the large displacement of $1-10 \mu \mathrm{C} / \mathrm{cm}^{2}$ in PVDF. 
The selected insulator should have a large permittivity connected with a high breakdown field. We chose $1.5 \mu \mathrm{m}$ thick PETP film (Hostaphan, reg.TM of Hoechst, Germany) for that purpose. The dielectric strength is calculated from the data sheet, given by the displacement at the breakdown field: $D=$ $\epsilon_{0} \epsilon E=1 \mu \mathrm{C} / \mathrm{cm}^{2}$.

The sample material is a copolymer of PVDF with TrFE, which has been annealed 2 hours at $135^{\circ} \mathrm{C}$.

\section{Results}

Figure 2 shows a comparison of the dielectric displacement measured with the capacitor $C_{m}$ (comp. Fig. 1) of two poling processes with and without insulating layers at the electrodes, respectively.

The applied potential was adjusted in order to compensate for the voltage drop at the insulators and to obtain the same diplacement.

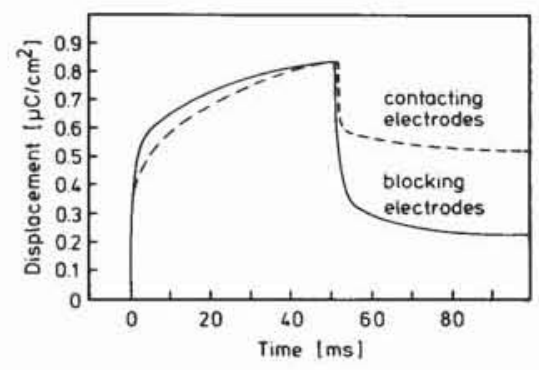

Figure 2: Dielectric displacement measured with capacitor $C_{m}$. The high voltage was applied for $50 \mathrm{~ms}$. The dotted line corresponds to the poling experiment with metal contacts. The full drawn line corresponds to the experiment with blocking electrodes.

The high voltage is applied for $50 \mathrm{~ms}$. This time is well below the charge relaxation times of the blocking insulator $\tau_{i} \approx 10^{5} \mathrm{~s}$ and the PVDF sample $\tau_{p} \approx 100 \mathrm{~s}$. The dielectric displacement is constant in the sample sandwich under this condition. The sample is kept under short circuit condition after the high voltage impulse (compare for the experimental details of the high voltage pulse generator used [8] and the paper about "Dynamics of the Polarization in P(VDF-TrFE) Copolymers" in this volume).

The displacement and thus the degree of dipole orientation is equal in both cases. But the short time remanent polarization, which can be deduced from the displacement value after the high voltage impulse is significantly smaller in the case of blocking electrodes. After a few minutes of short circuit condition the polarization distribution in the film depth is measured using the pressure step technique (PPS) [7]. The corresponding results shows Figure 3.

The experiment shows that the resulting remanent polarization after a few minutes is about two orders of magnitude smaller if blocking electrodes are 


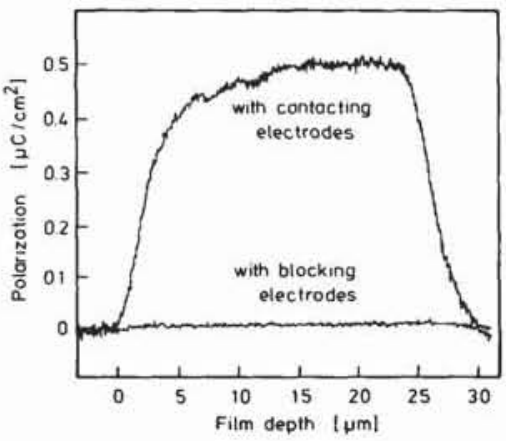

Figure 3: Polarization distribution in the film depth measured with the pressure step technique (PPS). The dotted line corresponds to the poling experiment with metal contacts. The full drawn line corresponds to the experiment with blocking electrodes.

used as compared to the ordinary poling with metal contacts.

Since for each experiment a new sample has to be used the observed reduction factors scatter due to differences in the morphology and preparation of the sample sandwich. For a series of 9 experiments with varying impulse length between 10 and $1000 \mathrm{~ms}$ we find reduction factors with a mean value of 16 . Figure 4 shows the data of this series.

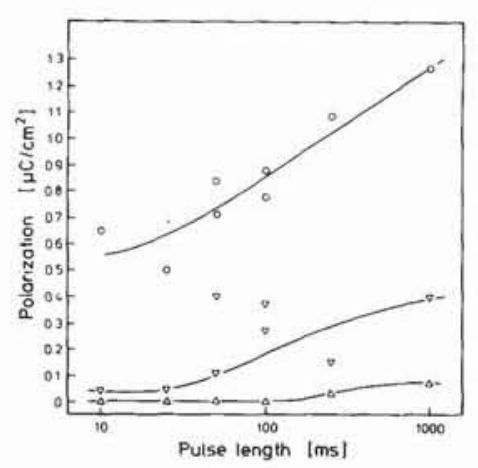

Figure 4: Polarization values depending on the poling impulse duration. $\mathrm{O}$ : maximum value of the dielectric displacement $d u$ ring field application, $\nabla$ : remanent polarization after poling with contacting metal electrodes, $\Delta$ : remanent polarization after poling with blocking electrodes. 


\section{Discussion}

The results of the poling experiments with blocking electrodes show that charge injection is a necessary condition for the stabilization of the polarization in PVDF and its copolymer with TrFE. The anisotropy field of the near-hexagonal lattice of the polar $\beta$-phase is not strong enough to account for the long time stability of the polarization and the high coercitive field.

An additional interaction between the injected charges and the dipolar polarization of the polar crystallites can describe the observed behavior.

At the surface of the polar crystallites, where the molecular dipoles end, coulomb traps are formed. The injected charges become deeply trapped. The dipole orientation in the crystallites are fixed in the electric field of the trapped charges. There is no residual electric field in the material, because the trapped charges compensate the polarization of the crystallites. Figure 5 shows a section of a $\beta$-crystallite perpendicular to the chain direction.

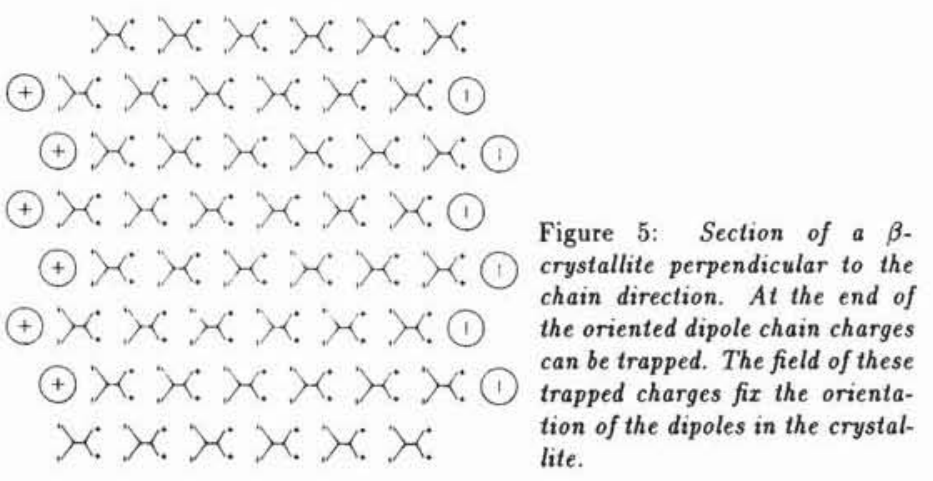

Under the condition of perfectly blocking electrodes it is expected that no remanent polarization can develop at all by poling the films, because not enough charges are available to fix the dipole orientation. The observed small remanent polarization could be either stabilized by intrinsic charges or by charges, which were able to pass the blocking insulator. Since the blocking insulator is strongly stressed near its breakdown field it can be assumed that charge injection transport in the insulator can account for the observed small remanent polarization in the $\mathrm{P}(\mathrm{VDF}-\mathrm{TrFE})$ samples. This is confirmed by measurements of the charge density profile inside the insulator after the poling experiments. We find a charge density in the insulator film of about the same order of magnitude as necessary to compensate the remanent polarization in the $\mathrm{P}(\mathrm{VDF}-\mathrm{TrFE})$ sample. 


\section{Summary}

The poling of PVDF with blocking electrodes hinders the charge injection into the material. Compared to samples, which have been poled with metal contacts, the resulting remanent polarization is more than one order of magnitude smaller when poled with blocking electrodes.

This behavior cannot be explained by a ferroelectric model for the polar crystallites alone.

The stabilization of the polarization is from our experimental results connected with the injection of charges and charge trapping at the crystallites surface.

We gratefully acknowledge financial support by the Deutsche Forschungsgemeinschaft.

\section{References}

[1] R. G. Kepler, R. A. Anderson, "Piezoelectricity in Polymers", CRC Critical Reviews in Solid State and Materials Science, Vol.9, 399-447, 1980.

[2] G. Pfister, M. Abkowitz, R. G. Crystal, J. Appl. Phys., Vol.44, 2064-2071, 1973.

[3] N.Murayama, J. Polym. Sci., Polym. Phys., Vol. 13, 929-946, 1975.

[4] H. Sussner, K. Dransfeld, J. Polym. Sci., Polym. Phys., 16, 529-542, 1978.

[5] R.G. Kepler, R.A. Anderson, J. Appl. Phys., Vol.49, 1232-1235, 1978.

[6] M. G. Broadhurst, G. T. Davis, in G. M. Sessler (ed.), "Electrets", Springer 1980 und 1988

[7] W. Eisenmenger, M. Haardt, Sol. State Comm., Vol.41, 917-920, 1982.

[8] M. Womes, E. Bihler, W. Eisenmenger, IEEE Trans. El. Ins., Vol.24, 461468,1989 . 